\title{
MRI of "Diffusion" in the Human Brain: New Results Using a Modified CE-FAST Sequence
}

\author{
Klaus-Dietmar Merboldt, Harald Bruhn, Jens Frahm, \\ Michael L. Gyngell, WolfGang Hänicke, AND Michael Deimling* \\ Max-Planck-Institut für biophysikalische Chemie, Postfach 2841, D-3400 Göttingen, Federal Republic of \\ Germany; and * Bereich Medizinische Technik, Siemens AG, Henkestrasse 127, \\ D-8520 Erlangen, Federal Republic of Germany
}

Received August 19, 1988; revised November 29, 1988

\begin{abstract}
"Diffusion-weighted" MRI in the normal human brain and in a patient with a cerebral metastasis is demonstrated. The method employed was a modified CE-FAST sequence with imaging times of only 6-10 $\mathrm{s}$ using a conventional $1.5-\mathrm{T}$ whole-body MRI system (Siemens Magnetom). As with previous phantom and animal studies, the use of strong gradients together with macroscopic motions in vivo causes unavoidable artifacts in diffusion-weighted images of the human brain. While these artifacts are shown to be considerably reduced by averaging of $8-16$ images, the resulting diffusion contrast is compromised by unknown signal losses due to motion. (c) 1989 Academic Press, Inc.
\end{abstract}

\section{INTRODUCTION}

Magnetic resonance techniques for the study of molecular self-diffusion rely on the application of strong self-refocusing magnetic field gradients $(1,2)$. However, the method does not measure the self-diffusion coefficient directly but the signal attenuation due to a net positional displacement of spins achieved within a chosen "diffusion" time. Thus, in vivo studies of incoherent diffusional processes are severely compromised by competitive contributions from microcirculation (perfusion), macroscopic motions (tissue pulsations, breathing, patient movements, system instabilities), and macroscopic fluid flow. Typical properties of these processes are summarized in Table 1. Accordingly, a separation of coherent and incoherent processes, although in principle possible using motion-rephasing gradients (3), does not correlate with a separation of diffusion and perfusion. The importance of relative contributions from coherent and incoherent capillary blood flow has been discussed in Ref. (4). Access to perfusion is further complicated by the low percentage of spins involved (brain) and the much stronger signal attenuation observed due to macroscopic motions. The influence of slow flow and pulsatile motion on diffusionweighted MR images has been investigated in a previous study on phantoms and rabbit brains in vivo (5). The investigations were performed using the CE-FAST sequence (6-8) modified to allow rapid MRI of diffusion $(9,10)$.

In this communication we describe applications of the modified CE-FAST sequence to studies of the human brain using a conventional 1.5-T whole-body MRI 
TABLE 1

Typical Properties of Transport Processes and Motion in Vivo That Give Rise to Mean Positional Displacements Measured Using Diffusion-Type MR Sequences

\begin{tabular}{|c|c|c|c|}
\hline Process & Character & $\begin{array}{c}\text { Displacement } \\
(100 \mathrm{~ms}) \\
(\mu \mathrm{m})\end{array}$ & $\begin{array}{c}\text { Spins } \\
\text { involved } \\
(\%)\end{array}$ \\
\hline Diffusion & Incoherent & 10 & 100 \\
\hline Perfusion (brain) & Incoherent/coherent & 100 & $2-5$ \\
\hline Macroscopic motions & Coherent/incoherent & 100 & 100 \\
\hline Macroscopic flow & Coherent & $>1000$ & 100 \\
\hline
\end{tabular}

Note. Macroscopic motions refer to tissue pulsations, breathing, general patient movements, and dynamic system instabilities.

system. In the absence of motion-rephasing waveforms for the additional "diffusion" gradients, all processes shown in Table 1 give rise to signal losses depending on their mean positional displacement. It is demonstrated that weak gradients are sufficient to remove all signal contributions from perfusion as well as from incoherent macroscopic motions, whereas coherent macroscopic motions cause artifacts (ghosting) in the phase-encoding dimension of individual diffusion-weighted brain images. These artifacts may be effectively reduced by averaging of subsequently acquired images. Macroscopic flow prevents the establishment of steady-state conditions (11) and, therefore, is not seen in CE-FAST images. The contrast observed in heavily diffusionweighted images is determined by a combination of true diffusion contrasts and unknown signal losses due to averaging of macroscopically moving spins. Because of this and the complex nature of the SSFP echo signal we explicitly avoid the calculation of meaningless diffusion coefficients from images obtained at different gradient strengths.

\section{METHODS}

The modification of the CE-FAST sequence for diffusion-weighted MRI involves the incorporation of a diffusion gradient pulse in each repetition interval. This extra gradient pulse may be applied in the direction of the slice selection or frequencyencoding gradient or both. It is refocused by the fact that the CE-FAST sequence acquires the echo part rather than the FID of the steady-state free precession (SSFP) signal. For acquisition of the SSFP echo preceding the radiofrequency (RF) pulses its position is shifted to $10 \mathrm{~ms}$ in advance of the RF pulses by reversal of the read gradient. Detailed descriptions of the basic sequence (6-8) and the diffusionweighted version used (10) are given elsewhere. Since "diffusion-weighting" in vivo does not automatically lead to pure diffusion contrast, this term is used only as a terminus technicus describing the use of a pair of self-refocusing gradients as conventionally employed for diffusion studies. Informed consent of the volunteers and the patient was obtained prior to the investigations. 


\section{RESULTS AND DISCUSSION}

Diffusion imaging studies of the brain were carried out on healthy volunteers and on a patient using a conventional 1.5-T whole-body MRI system (Siemens Magnetom). The maximum available gradient strength was $10 \mathrm{mT} \mathrm{m}^{-1}$. Motion artifacts were observed in all single-excitation CE-FAST images recorded at high diffusion gradient strengths. This is demonstrated in Fig. 1 by a series of 10-s images ( $\mathrm{TR}=40 \mathrm{~ms}$ ) obtained using diffusion gradients (16 ms duration) of $1.0(\mathrm{a}), 7.0$ (b), and $9.8 \mathrm{mT} \mathrm{m}^{-1}(\mathrm{c}, \mathrm{d})$. The occurrence of smearing and ghosting artifacts in the phase-encoding dimension of the images is independent of both the particular MRI system and the sequence used. It is a general experimental finding that in contrast to phantom studies $(12,13)$ diffusion-weighted images of animals and humans in vivo become degraded by motion artifacts. In fact, even ECG synchronization is unable to remove artifacts and signal losses due to in-scan motions during the diffusion time, i.e., between the applied self-refocusing diffusion gradients. In the present study the extent of image degradation varied considerably between different individuals. Image (d) in Fig. 1 represents the average of eight subsequent scans recorded in a total measuring time of about $80 \mathrm{~s}$. Obviously, motion artifacts can be sufficiently reduced by averaging of 8-16 images. However, this is achieved at the expense of signal losses depending on the extent of motion artifacts in single-excitation images. Averaging of sequentially recorded image data turned out to be superior to direct averaging of data from individual phase-encoding steps.

Diffusion-weighted brain images of two healthy volunteers are shown in Fig. 2. Images $(\mathrm{a}-\mathrm{c})$ of the first volunteer refer to a weak diffusion-weighting as represented by the time integral of the respective gradient pulses ( 5 ms duration, 1.0 (a), 7.0 (b), and $9.8 \mathrm{mT} \mathrm{m}^{-1}$ (c) strength). The images were recorded within an overall measuring time of about $1.5 \mathrm{~min}$ ( TR $=23 \mathrm{~ms}, 16$ acquisitions) using a flip angle of $75^{\circ}$. The signal intensity from CSF becomes strongly attenuated by macroscopic flow and pulsatile motions even in the presence of a diffusion-weighting that is weak enough to leave signals from normal brain almost unaffected. This finding is in agreement with a previous observation of the strong sensitivity of SSFP signals to slow flow (14). A change of the orientation of the diffusion gradient from the slice selection direction to the frequency-encoding direction (horizontal axis in Fig. 2) resulted in a slower decrease of the CSF signal with gradient strength. This finding is probably due to the preferred flow direction of CSF.

Images (d)-(f) of the second volunteer in Fig. 2 were obtained with much stronger diffusion weighting. Using the same gradient strengths as in (a)-(c) the duration of the gradient pulses was increased to $20 \mathrm{~ms}$. Consequently, the repetition time had to be increased to $\mathrm{TR}=40 \mathrm{~ms}$ yielding measuring times of about $1.5 \mathrm{~min}$ for eight averages. In the resulting images the signal intensity of gray matter decreased more than that of white matter. However, contrast in Fig. $2 \mathrm{f}$ is not only due to true differences in the molecular self-diffusion coefficients of water in gray and white matter but also affected by motion averaging as discussed in Fig. 1. Although image artifacts due to motion may be eliminated by averaging, the diffusion-weighted images are influenced and possibly dominated by macroscopic motions. Motion averaging leads to signal losses which may be spatially heterogeneous. For example, since brain pulsa- 


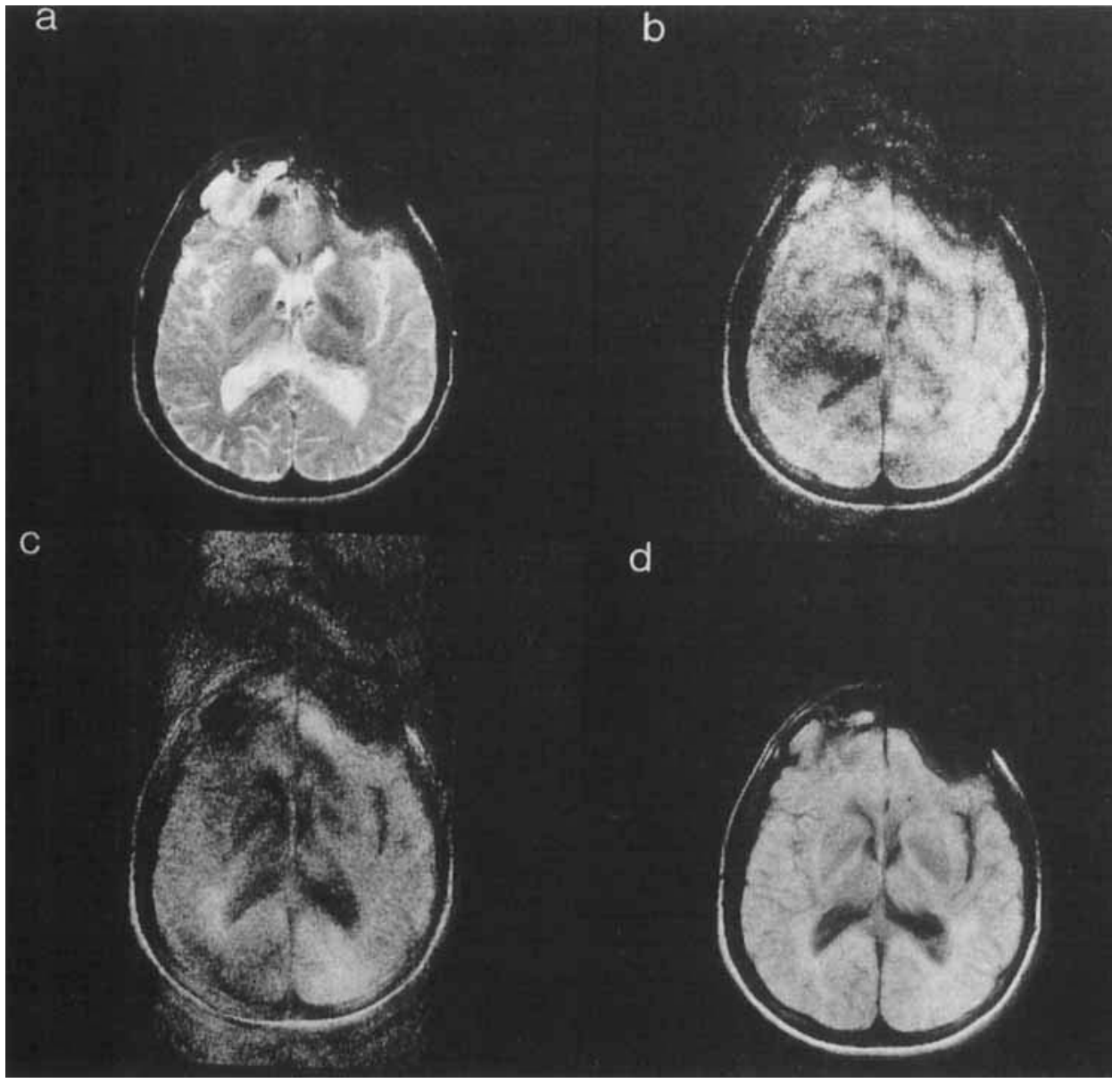

FIG. 1. Sixty-four-megahertz (1.5-T) "diffusion-weighted" CE-FAST images $(256 \times 256$ pixel resolution, flip angle $75^{\circ}$ ) of the brain of a healthy volunteer. The images were recorded using diffusion gradient strengths of $1.0(\mathrm{a}), 7.0(\mathrm{~b})$, and $9.8 \mathrm{~m}^{\prime} \mathrm{m} \mathrm{m}^{-1}(\mathrm{c}, \mathrm{d})$, respectively. Images (a)-(c) represent single excitation images corresponding to measuring times of about $10 \mathrm{~s}(\mathrm{TR}=40 \mathrm{~ms})$. Image $(\mathrm{d})$ represents the average of eight subsequent scans leading to a total measuring time of about $80 \mathrm{~s}$. All images $(10 \mathrm{~mm}$ slice thickness) were obtained using diffusion gradients (frequency-encoding direction) of 16 ms duration.

tions expand radially the degree of imposed "motion contrast" may be different for gray and white matter due to structural differences and location.

Figure 3 depicts transaxial diffusion-weighted CE-FAST images of a 72-year-old female patient with a cerebral metastasis of a hypernephroma in the left occipital area. Each image was recorded within a measuring time of about 1 min using eight acquisitions. A weak diffusion-weighting was employed in images (a) and (b) using a repetition time of $T R=25 \mathrm{~ms}$ and gradient pulses of $5 \mathrm{~ms}$ duration and 1.0 and $7.0 \mathrm{mT} \mathrm{m}^{-1}$ strength. An intermediate diffusion-weighting was obtained in images (c) and (d) using TR $=29 \mathrm{~ms}$ and gradient pulses of $9 \mathrm{~ms}$ duration and 7.0 and 9.8 


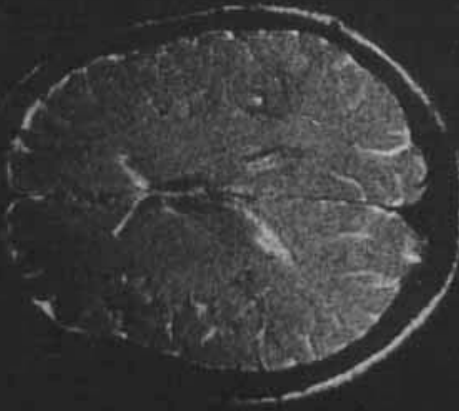

()

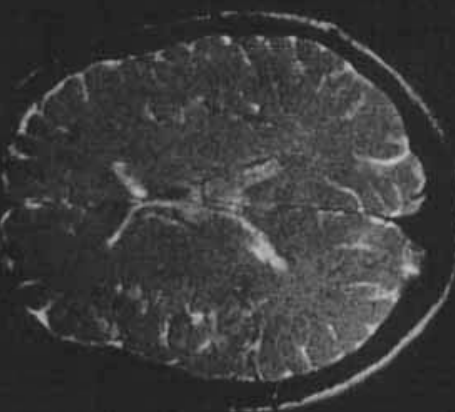

(9)

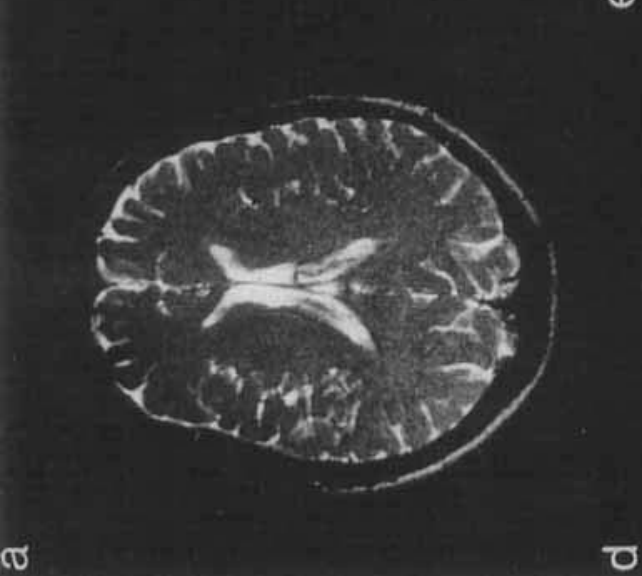

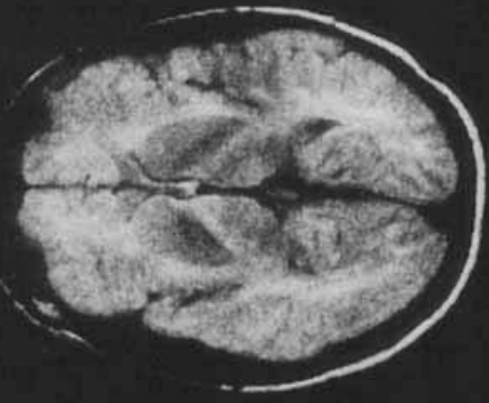

4

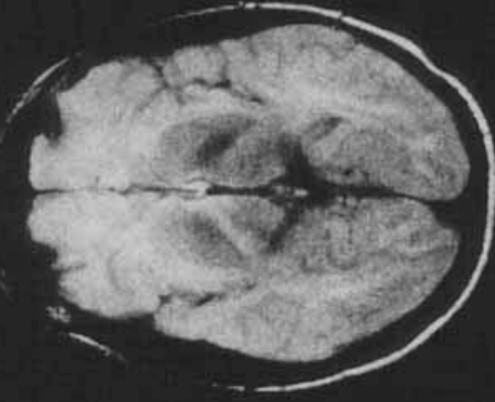

(1)

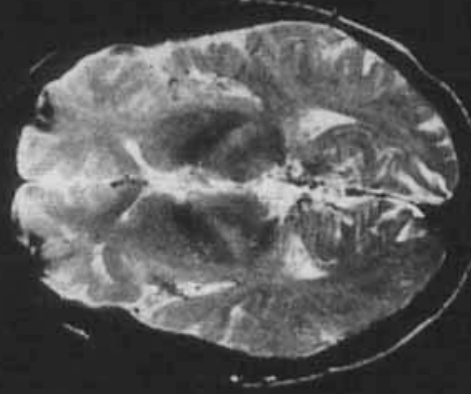

宏焉

政

쿵

要递

要焉

过品

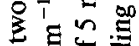

눙ㅇㅇ

है

ํํㅇ

氖苋

응횽

in 0

क웡

굥

응

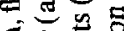

드을 해앴

要得

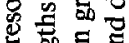

하을

希氖

北

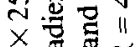

总唯

ปู है है

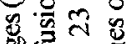

"I

政 존

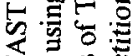

红 .5 过

讨要焉

in

总吉总

की

我舟

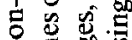

要

进焉焉

是

$F$ के

n'

可

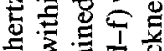

要

兵

妾要击

的

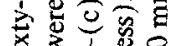

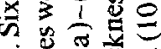

$\sim$ 尊

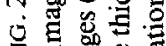

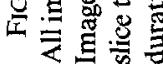




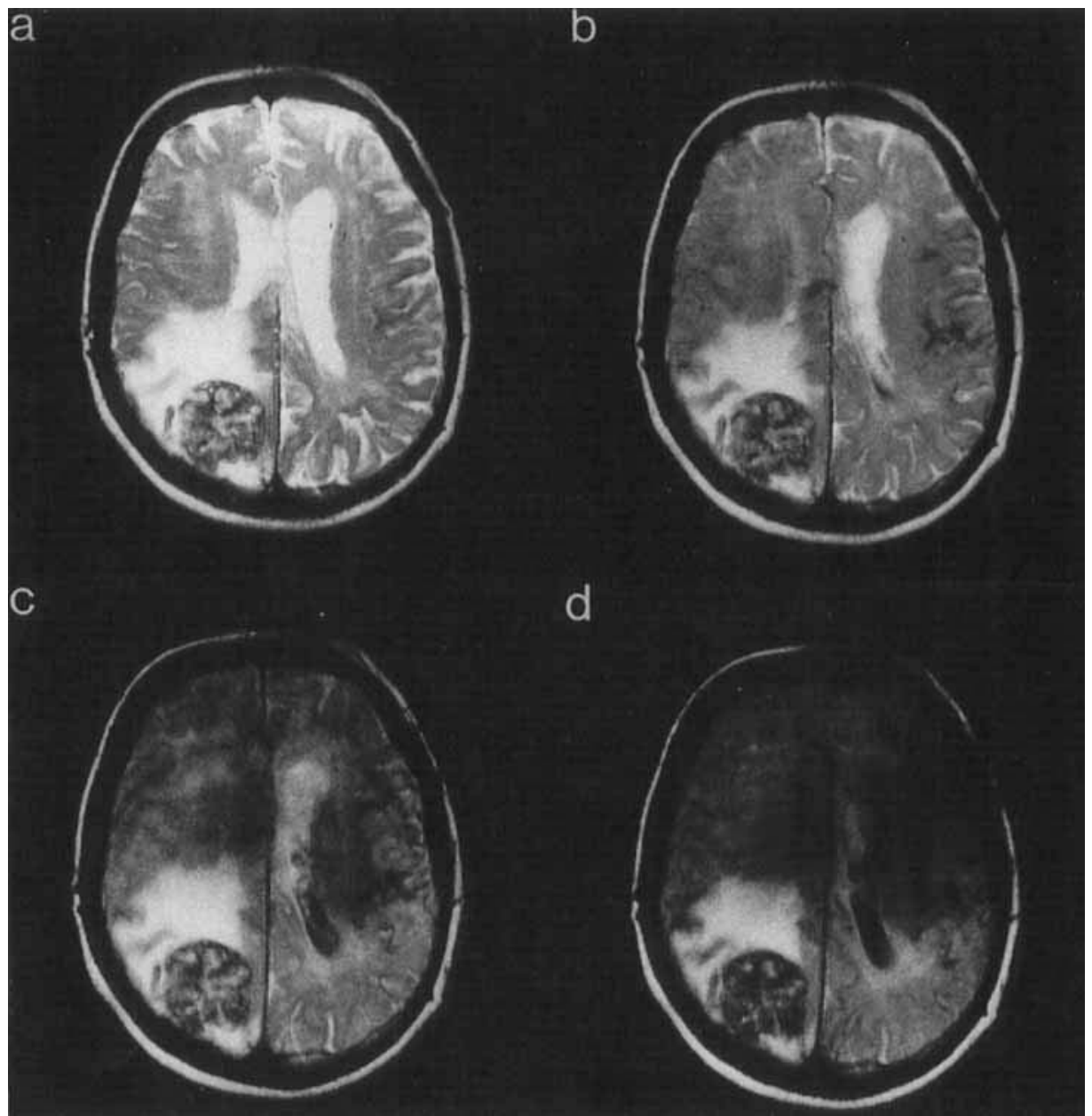

FIG. 3. Sixty-four-megahertz (1.5-T) "diffusion-weighted" CE-FAST images of a 72-year-old female patient with a cerebral metastasis of a hypernephroma in the left occipital area. Each image $(256 \times 256$ pixel resolution, $10 \mathrm{~mm}$ slice thickness) was recorded within a measuring time of about $1 \mathrm{~min}$ using eight acquisitions. Images ( $\mathrm{a}$ ) and ( $\mathrm{b}$ ) were obtained using repetition times of TR $=25 \mathrm{~ms}$ and diffusion gradients (frequency-encoding direction) of $5 \mathrm{~ms}$ duration and strengths of 1.0 and $7.0 \mathrm{mT} \mathrm{m}^{-1}$, respectively. Images (c) and (d) were obtained using TR $=29 \mathrm{~ms}$ and gradients of $9 \mathrm{~ms}$ duration and strengths of 7.0 and 9.8 $\mathrm{mT} \mathrm{m}^{-1}$, respectively.

$\mathrm{mT} \mathrm{m}^{-1}$ strength. As in the volunteer studies the strong signal attenuation of CSF by relatively weak gradient pulses (Figs. $3 \mathrm{~b}$ and $3 \mathrm{c}$ ) is attributed to macroscopic flow effects. A further increase of the diffusion-weighting in Fig. $3 \mathrm{~d}$ results in a total loss of signal intensities from CSF, whereas the edema surrounding the cerebral metastasis remains bright. This may be explained by the absence of macroscopic flow in the edema. Even for a high molecular mobility of water within the edema and a selfdiffusion coefficient close to that of bulk water the applied diffusion-weighting is only 
comparable to a value as used for Figs. $2 \mathrm{~d}$ and $2 \mathrm{e}$. It is, therefore, not strong enough to cause significant signal attenuation of the edema or to provide gray/white matter contrast as in Fig. 2f. The signal loss observed in the front of the head (Figs. $3 \mathrm{c}$ and 3d) may have resulted from patient movements. For a supine position related spatial displacements are more pronounced in the front of the head than at the back. In general, diffusion-weighted CE-FAST images allow an easy and relatively rapid discrimination of edema and CSF-containing structures on the basis of the macroscopic mobility of water.

\section{CONCLUSIONS}

Magnetic resonance imaging of diffusion in vivo is severely impeded by spatial misregistration artifacts. These are due to slow flow, tissue pulsations, and unavoidable patient movements and are dramatically increased by the use of strong magnetic field gradient pulses as required for diffusion weighting. It is demonstrated that respective image artifacts are considerably reduced by averaging data of subsequently recorded images. However, the resulting contrast as, e.g., seen between gray and white matter in normal volunteers, represents a mixture of "true" diffusion contrasts and unknown signal losses due to motion averaging. In these circumstances any computation of diffusion coefficients must remain ambiguous. Using only moderate diffusionweighting excellent discrimination among CSF, edema, and tumorous tissue was obtained in a patient with a cerebral metastasis.

\section{ACKNOWLEDGMENTS}

Financial support by the Bundesminister für Forschung und Technologie (BMFT) of the Federal Republic of Germany (Grant 0 I VF 8606/6) is gratefully acknowledged. We thank Dr. Hamburger, Department of Neurosurgery, Klinikum Grosshadern in Munich, for allowing us to study his patient.

\section{REFERENCES}

1. E. O. STEJSKal and J. E. TANner, J. Chem. Phys. 42, 288 (1965).

2. J. E. TANner, J. Chem. Phys. 52, 2522 (1970).

3. P. M. Pattany, J. J. Phillips, L. C. Chiu, J. L. Duerk, J. M. McNally, and S. N. Mohapatra, J. Comput. Assist. Tomogr. 11, 369 (1987).

4. D. Le Bihan, E. Breton, D. Lallemand, M. L. Aubin, J. Vignaud, and M. Laval-Jeantet, Radiology 168, 497 (1988).

5. K. D. Merboldt, W. Hänicke, M. L. Gyngell, J. Frahm, and H. Bruhn, submitted for publication.

6. R. C. Hawkes and S. PATZ, Magn. Reson. Med. 4, 9 (1987).

7. M. L. GYNGELL, Magn. Reson. Imaging 6, 415 (1988).

8. M. L. GYNGELL, J. Magn. Reson., in press.

9. D. Le Bihan, Magn. Reson. Med. 7, 346 (1988).

10. K. D. Merboldt, W. Hänicke, M. L. Gyngell, J. Frahm, and H. Bruhn, J. Magn. Reson., in press.

11. E. K. Fram, J. P. Karis, A. Evans, A. Shimakawa, G. A. Johnson, And R. F. Herfkens, "Society of Magnetic Resonance in Medicine, 6th Annual Meeting, August 17-21, 1987, New York," p. 314.

12. K. D. MERBOLDT, W. HÄNICKE, AND J. FrahM, J. Magn. Reson. 64, 479 (1985).

13. K. D. Merboldt, W. Hänicke, ANd J. FrahM, Ber. Bunsenges. Phys. Chem. 91, 1124 (1987).

14. S. PATZ AND R. C. HaWkes, Magn. Reson. Med. 3, 140 (1986). 